\title{
A tractable inhomogeneous closure theory for flow over mean topography
}

\author{
T. J. O'Kane* $\quad$ J. S. Frederiksen ${ }^{\dagger}$
}

(Received 8 August 2003, revised 10 December 2003)

\begin{abstract}
The quasi-diagonal direct interaction approximation (QDIA) is shown to be a computationally tractable closure theory for inhomogeneous two-dimensional turbulent flow over mean (single-realization) topography. In this paper numerical results for the QDIA are compared to direct numerical simulation (DNS) at moderate Reynolds number for two cases with quite different topographic and mean field amplitudes. The QDIA is found to be in excellent agreement with DNS for cases where the small-scale topographic amplitude is significant. For cases where the small-scale topography is weak, the QDIA closely reproduces the evolving mean field and large-scale energy containing transients but under represents the amplitudes of the small-scale transients in
\end{abstract}

${ }^{*}$ CSIRO Atmospheric Research, Aspendale, Australia. mailto:Terence.0'Kane@csiro.au

${ }^{\dagger}$ CSIRO Atmospheric Research, Aspendale, Australia. mailto: Jorgen. Frederiksen@csiro.au

See http://anziamj.austms.org.au/V45/CTAC2003/Okan/home.html for this article, (c) Austral. Mathematical Soc. 2004. Published April 3, 2004. ISSN 1446-8735 
a similar way to the homogeneous DIA. We discuss the prospects of ameliorating the small-scale deficiencies using a regularization of the interaction coefficients.

\section{Contents}

1 Introduction

C136

2 The QDIA closure for 2-D turbulent flow over general topography

C138

3 Moderate Reynolds number turbulence

C142

4 Discussion and Conclusions

C146

References

C147

\section{Introduction}

The direct interaction approximation (DIA) [1] represents a major advance on the path to developing a tractable statistical closure for fluid turbulence. The isotropic Eulerian DIA is a second-order renormalized perturbation theory in which the propagators, namely the two-point cumulant and response function, are renormalized while the vertex term is bare and equal to the interaction coefficient [2]. An unfortunate consequence of the bare vertex approximation is that the DIA does not adequately describe the convection of small-scale eddies by large eddies. At very high Reynolds number this leads to the DIA predicting a $k^{-5 / 2}$ rather than a $k^{-3}$ enstrophy inertial range $[3,4]$ for two dimensional turbulence. Nevertheless the DIA is in good agreement with direct numerical simulations (DNS) in the energy containing 
range of large scales and contains no arbitrary parameters, unlike the eddydamped quasi-normal Markovian model (EDQNM) [15] and test-field model (TFM) [5, 4]. The DIA is less restrictive its applications than Markovian closures but is computationally more expensive because of the potentially long time-history integrals.

The incorporation of the statistical properties of random topography into homogeneous closure models was achieved in two important papers by Herring [8] and Holloway [9] using DIA and TFM models. These papers generated considerable insight into how the statistical properties of random topography determine the spectra of transient vorticity variance but have left open the question of how the mean single realization topography of oceans and atmospheres determines the structures of the mean flows. Kraichnan formulated a DIA closure theory for general inhomogeneous flows [10] and noted that the inhomogeneous DIA was not computationally tractable without some form of diagonalization [11]. Recently, Frederiksen [12] developed a quasi-diagonal DIA closure, which he applied to barotropic flow over topography, by expressing the off-diagonal elements of the covariance and response function matrices in terms of the diagonal elements. In a previous low resolution study of the QDIA closure O'Kane \& Frederiksen [16] introduced a generalized cumulant update method for inhomogeneous flows, in which the potentially long time-history integrals are periodically truncated and the two- and three-point cumulants calculated and used in the new non-Gaussian initial conditions of the restart procedure.

The objectives of this present paper are threefold. The first is to demonstrate that the inhomogeneous quasi-diagonal QDIA is not only computationally tractable but that it gives results consistent with previous homogeneous statistical closure studies. The second objective is to examine the effect of various specifications of the topography and strengths of the mean fields on the accuracy of the QDIA. Thirdly we wish to extend the results of our earlier very low resolution study (O'Kane \& Frederiksen [16]) to significantly higher resolutions. Our aim is to gain insight into the validity of the quasi-diagonal 
approximation and, given that the formal theoretical treatment assumes weak topography and weak mean field prior to renormalization, to ascertain the effect of strong topographies and mean fields. We are also interested to see whether the spurious convection effects associated with the DIA treatment of the three-point term $[13,14]$ are increased or decreased as the topographic amplitude is increased in the small scales.

In Section 2 we begin by defining the barotropic vorticity equation for flow over topography and then summarize the QDIA closure. In Section 3 we present a comparison of the QDIA and DNS for circularly truncated $k=48$ resolution (C48) at moderate large scale Reynolds number $\left(R_{L} \approx 300\right)$ and for two different specifications of the topography and initial mean fields. In the first case the topographic amplitude scales like $1 / k$ and results in a strong initial mean field at the smallest scales. In the second case the topographic amplitude scales like $1 / k^{2}$ and gives a weak initial mean field at the smallest scales. As for previous isotropic studies $[6,7,14]$ both DNS and closure models have been formulated for discrete wavenumbers. This allows us to make direct comparison without the need to account for systematic differences arising from the more traditional logarithmic discretization schemes and continuous wavenumbers $[13,15]$ commonly employed in closure models to reach high wavenumbers. Our conclusions appear in Section 4.

\section{The QDIA closure for 2-D turbulent flow over general topography}

The evolution of two-dimensional decaying flow over a fixed topography on a periodic $f$-plane $(0 \leq x \leq 2 \pi),(0 \leq y \leq 2 \pi)$ is described by the nondimensional barotropic vorticity equation, which takes the form

$$
\frac{\partial \zeta}{\partial t}=-J(\psi, \zeta+h)+\hat{\nu} \nabla^{2} \zeta
$$


Here $\hat{\nu}$ is the bare viscosity, $J(\psi, \zeta)$ the Jacobian and the vorticity is the Laplacian of the stream-function $\zeta=\nabla^{2} \psi$. We may write Eq. (1) in spectral form via the standard Fourier transform relationships. Thus the spectral vorticity equation takes the form

$$
\begin{aligned}
& \left(\frac{\partial}{\partial t}+\hat{\nu} k^{2}\right) \zeta_{\mathbf{k}}(t)=\sum_{\mathbf{p}} \sum_{\mathbf{q}} \delta(\mathbf{k}+\mathbf{p}+\mathbf{q}) \\
& \quad \times\left[K(\mathbf{k}, \mathbf{p}, \mathbf{q}) \zeta_{-\mathbf{p}} \zeta_{-\mathbf{q}}+A(\mathbf{k}, \mathbf{p}, \mathbf{q}) \zeta_{-\mathbf{p}} h_{-\mathbf{q}}\right]
\end{aligned}
$$

where $\mathbf{k}=\left(k_{x}, k_{y}\right), k=\left(k_{x}^{2}+k_{y}^{2}\right)^{1 / 2}$ and $\zeta_{-\mathbf{k}}$ is conjugate to $\zeta_{\mathbf{k}}$. The interaction coefficients are governed by the following relationships

$$
\begin{aligned}
& A(\mathbf{k}, \mathbf{p}, \mathbf{q})=-\left(p_{x} q_{y}-p_{y} q_{x}\right) / p^{2}, \\
& K(\mathbf{k}, \mathbf{p}, \mathbf{q})=\frac{1}{2}\left(p_{x} q_{y}-p_{y} q_{x}\right)\left(p^{2}-q^{2}\right) / p^{2} q^{2}, \\
& \text { where } \delta(\mathbf{k}+\mathbf{p}+\mathbf{q})= \begin{cases}1, & \text { if } \mathbf{k}+\mathbf{p}+\mathbf{q}=\mathbf{0}, \\
0, & \text { otherwise. }\end{cases}
\end{aligned}
$$

For an ensemble of flows satisfying Eq. (2), we can express the vorticity $\zeta_{\mathbf{k}}=\left\langle\zeta_{\mathbf{k}}\right\rangle+\hat{\zeta}_{\mathbf{k}}$ where \langle\rangle denotes the mean and ${ }^{\wedge}$ denotes the deviation from the ensemble mean. The two-time two-point cumulant $C_{\mathbf{k}-\mathbf{l}}(t, \hat{t})=\left\langle\hat{\zeta}_{\mathbf{k}}(t) \hat{\zeta}_{-\mathbf{l}}(\hat{t})\right\rangle$, the response to infinitesimal perturbations $R_{\mathbf{k} \mathbf{l}}(t, \hat{t})=\left\langle\delta \hat{\zeta}_{\mathbf{k}}(t) / \delta \hat{f}_{\mathbf{l}}^{0}(\hat{t})\right\rangle$ and we use the shorthand notation $C_{\mathbf{k}}=C_{\mathbf{k}-\mathbf{k}}$ and $R_{\mathbf{k}}=R_{\mathbf{k k}}$ for the diagonal terms. The two-time cumulant can then be expressed in terms of two- and three-point terms

$$
\begin{aligned}
& \left(\frac{\partial}{\partial t}+\hat{\nu} k^{2}\right) C_{\mathbf{k}}(t, \hat{t})=\sum_{\mathbf{p}} \sum_{\mathbf{q}} \delta(\mathbf{k}+\mathbf{p}+\mathbf{q}) A(\mathbf{k}, \mathbf{p}, \mathbf{q}) C_{-\mathbf{p}-\mathbf{k}}(t, \hat{t}) h_{-\mathbf{q}} \\
& +\sum_{\mathbf{p}} \sum_{\mathbf{q}} \delta(\mathbf{k}+\mathbf{p}+\mathbf{q}) K(\mathbf{k}, \mathbf{p}, \mathbf{q}) \\
& \quad \times\left[\left\langle\zeta_{-\mathbf{p}}(t)\right\rangle C_{-\mathbf{q}-\mathbf{k}}(t, \hat{t})+C_{-\mathbf{p}-\mathbf{k}}(t, \hat{t})\left\langle\zeta_{-\mathbf{q}}(t)\right\rangle+\left\langle\hat{\zeta}_{-\mathbf{p}}(t) \hat{\zeta}_{-\mathbf{q}}(t) \hat{\zeta}_{-\mathbf{k}}(\hat{t})\right\rangle\right]
\end{aligned}
$$


Similarly the equation for the mean-field depends on the two-point singletime cumulant

$$
\begin{aligned}
& \left(\frac{\partial}{\partial t}+\hat{\nu} k^{2}\right)\left\langle\zeta_{\mathbf{k}}(t)\right\rangle= \\
& \sum_{\mathbf{p}} \sum_{\mathbf{q}} \delta(\mathbf{k}+\mathbf{p}+\mathbf{q})\left\{K(\mathbf{k}, \mathbf{p}, \mathbf{q})\left[\left\langle\zeta_{-\mathbf{p}}(t)\right\rangle\left\langle\zeta_{-\mathbf{q}}(t)\right\rangle+C_{-\mathbf{p}-\mathbf{q}}(t, t)\right]\right. \\
& \left.\quad+A(\mathbf{k}, \mathbf{p}, \mathbf{q})\left\langle\zeta_{-\mathbf{p}}(t)\right\rangle h_{-\mathbf{q}}\right\}
\end{aligned}
$$

Frederiksen [12] used renormalized perturbation theory to close these equations by expressing the two- and three-point cumulants and the twopoint response function in terms of diagonal elements. Thus

$$
\begin{aligned}
C_{\mathbf{k}-\mathbf{l}}(t, \hat{t})= & \int_{t_{0}}^{t} d s R_{\mathbf{k}}(t, s) C_{\mathbf{l}}(s, \hat{t}) \\
& \times\left[A(\mathbf{k},-\mathbf{l}, \mathbf{l}-\mathbf{k}) h_{(\mathbf{k}-\mathbf{l})}+2 K(\mathbf{k},-\mathbf{l}, \mathbf{l}-\mathbf{k})\left\langle\zeta_{(\mathbf{k}-\mathbf{l})}(s)\right\rangle\right] \\
& +\int_{t_{0}}^{t} d s R_{-\mathbf{l}}(\dot{t}, s) C_{\mathbf{k}}(t, s) \\
& \times\left[A(-\mathbf{l}, \mathbf{k}, \mathbf{l}-\mathbf{k}) h_{(\mathbf{k}-\mathbf{l})}+2 K(-\mathbf{l}, \mathbf{k}, \mathbf{l}-\mathbf{k})\left\langle\zeta_{(\mathbf{k}-\mathbf{l})}(s)\right\rangle\right](7)
\end{aligned}
$$

and similarly the two-point two-time response function is

$$
\begin{aligned}
R_{\mathbf{k} \mathbf{l}}(t, \hat{t})= & \int_{\dot{t}}^{t} d s R_{\mathbf{k}}(t, s) R_{\mathbf{l}}(s, \dot{t}) \\
& \times\left[A(\mathbf{k},-\mathbf{l}, \mathbf{l}-\mathbf{k}) h_{(\mathbf{k}-\mathbf{l})}+2 K(\mathbf{k},-\mathbf{l}, \mathbf{l}-\mathbf{k})\left\langle\zeta_{(\mathbf{k}-\mathbf{l})}(s)\right\rangle\right]
\end{aligned}
$$

The isotropic component of the flow is contained in the three-point term and is dealt with using the direct interaction approximation following Kraich- 
nan [1]:

$$
\begin{aligned}
& \left\langle\hat{\zeta}_{-\mathbf{l}}(t) \hat{\zeta}_{(\mathbf{l}-\mathbf{k})}(t) \hat{\zeta}_{\mathbf{k}}(\hat{t})\right\rangle \\
& =2 \int_{t_{0}}^{t} d s K(\mathbf{k},-\mathbf{l}, \mathbf{l}-\mathbf{k}) C_{-\mathbf{l}}(t, s) C_{(\mathbf{l}-\mathbf{k})}(t, s) R_{\mathbf{k}}(\dot{t}, s) \\
& +2 \int_{t_{0}}^{t} d s K(-\mathbf{l}, \mathbf{l}-\mathbf{k}, \mathbf{k}) R_{-\mathbf{l}}(t, s) C_{(\mathbf{l}-\mathbf{k})}(t, s) C_{\mathbf{k}}(\dot{t}, s) \\
& +2 \int_{t_{0}}^{t} d s K(\mathbf{l}-\mathbf{k},-\mathbf{l}, \mathbf{k}) R_{(\mathbf{l}-\mathbf{k})}(t, s) C_{-\mathbf{l}}(t, s) C_{\mathbf{k}}(\dot{t}, s) .
\end{aligned}
$$

Finally the equation for the diagonal response function takes the form

$$
\begin{aligned}
& \left(\frac{\partial}{\partial t}+\hat{\nu} k^{2}\right) R_{\mathbf{k}}(t, \hat{t})=\int_{t}^{t} d s \sum_{\mathbf{p}} \sum_{\mathbf{q}} \delta(\mathbf{k}+\mathbf{p}+\mathbf{q}) R_{\mathbf{k}}(s, \hat{t}) \\
& \left\{4 K(\mathbf{k}, \mathbf{p}, \mathbf{q}) K(-\mathbf{p},-\mathbf{q},-\mathbf{k}) R_{-\mathbf{p}}(t, s) C_{-\mathbf{q}}(t, s)\right. \\
& \quad+R_{-\mathbf{p}}(t, s)\left[2 K(\mathbf{k}, \mathbf{p}, \mathbf{q})\left\langle\zeta_{-\mathbf{q}}(t)\right\rangle+A(\mathbf{k}, \mathbf{p}, \mathbf{q}) h_{-\mathbf{q}}\right] \\
& \left.\quad \times\left[2 K(-\mathbf{p},-\mathbf{k},-\mathbf{q})\left\langle\zeta_{\mathbf{q}}(s)\right\rangle+A(-\mathbf{p},-\mathbf{k},-\mathbf{q}) h_{\mathbf{q}}\right]\right\}
\end{aligned}
$$

with $R_{\mathbf{k}}(t, t)=1$ and $R_{\mathbf{k}}(t, \hat{t})=0$ for $t<\dot{t}$. These equations then form a closed system for the mean field and covariance and response functions.

The numerical strategies that we use to solve the DNS and QDIA closure equations are the same as previously described in O'Kane \& Frederiksen [16] and very similar to those described by [7]. Both the DNS and closure equations use the predictor-corrector scheme for time-integration and the closure equations use the trapezoidal rule to evaluate the time-history integrals. For both the DNS and closure equations the interaction coefficients are precalculated as dense single dimensional arrays and the fields, cumulants and response functions are calculated and stored for $\mathbf{k}$ in a half space, with complex conjugacy used to obtain the complete functions. The DNS results are averages over large numbers of realizations in which the initial conditions are sampled from multivariate Gaussian distributions with specified mean and variance. 


\section{Moderate Reynolds number turbulence}

In this section we consider moderate Reynolds number turbulence making direct comparisons with the previous isotropic turbulence studies of Herring et al. [4] and Frederiksen \& Davies [7]. Our initial transient enstrophy spectrum corresponds exactly to Spectrum B of Frederiksen \& Davies [7] and closely to Spectrum II of Herring et al. [4]. Thus we start with an initial (twice) enstrophy spectrum of

$$
C_{\mathbf{k}}(0,0)=1.8 \times 10^{-1} k^{2} \exp \left(-\frac{2}{3} k\right) .
$$

The initial mean enstrophy spectrum is defined by an equilibrium mean vorticity field

$$
\left\langle\zeta_{\mathbf{k}}(0)\right\rangle=\left\langle\zeta_{\mathbf{k}}^{e q}\right\rangle=-b h_{\mathbf{k}} \frac{k^{2}}{a+b k^{2}}
$$

with parameters $a$ and $b$ given in Table 1 being the same as Frederiksen \& Sawford [17] used to fit the large scales of meteorological flows. The DNS and closure use nondimensional time steps of 0.003 and 0.004 for Case I and Case II calculations respectively, and are integrated for 100 time steps at C48 (circularly truncated wavenumber space with $1 \leq k \leq 48$ ) resolution. The DNS spectra represent ensemble averages of 100 realizations with standard deviations (not shown) comparable to those in Figure 3 of Frederiksen \& Davies [7]. A nondimensional viscosity of $\hat{\nu}=0.0025$ gives an initial Reynolds number of $\approx 305$ for Spectrum B (as in [7]).

The diagnostic quantity we use to compare the QDIA to DNS are band averaged mean, transient and total kinetic energy $E(k)$ for which the individual components

$$
E_{\mathbf{k}}^{\text {Total }}(t)=E_{\mathbf{k}}^{\text {Trans }}(t)+E_{\mathbf{k}}^{\text {Mean }}(t)=\frac{1}{2} \frac{C_{\mathbf{k}}(t, t)}{k^{2}}+\frac{1}{2}\left\langle\zeta_{\mathbf{k}}(t)\right\rangle\left\langle\zeta_{-\mathbf{k}}(t)\right\rangle / k^{2},
$$

are averaged over wavenumber bands of unit width as for Eq. 16 of Frederiksen \& Davies [7]. As well we use the large-scale Reynolds number $R_{L}$ 
evaluated using the transient part of the field only (defined in Eqs. 14 of [7]). In each case a single realization mean topography $h_{\mathbf{k}}=\left|h_{\mathbf{k}}\right| \times\left(\cos \theta_{\mathbf{k}}+i \sin \theta_{\mathbf{k}}\right)$ with fixed specified random phase $\theta_{\mathbf{k}}$ is considered. We compare two cases with identical initial transient fields but with respective topographies for which $\left|h_{\mathbf{k}}\right|^{2}=4 k /\left(1+k^{3}\right)$ (Case I) and $\left|h_{\mathbf{k}}\right|^{2}=16 k^{2} /\left(1+k^{3}\right)^{2}$ (Case II) and consequently initial mean fields that strongly differ at the smallest scales. These two cases are intended to identify the effect of the strength of the topography at the small scales on the spectra of the mean and transient fields. These choices of topography provide tests of the accuracy of the closure with markedly different topographic amplitudes at the small scales, and consequently differing small-scale eddy-topographic interaction strengths.

The initial off-diagonal elements are zero and the initial conditions are Gaussian. We find that in the presence of topographic interaction the flow evolves more rapidly and therefore show results at final times $t_{f}^{\text {CaseI }}=0.3$ or $t_{f}^{\text {CaseII }}=0.4$ compared to $t_{f}=0.8$ in the isotropic calculations of Frederiksen \& Davies [7]. The rapid evolution of the flow fields can be attributed to the presence of both topography and a mean-field forcing of the small-scale transients.

First consider Case I. In Figure 1(a) the initial mean and transient spectra are displayed with parameter values specified in Table 1. At the nondimensional time of $t_{f}=0.3$ the evolved QDIA mean and transient kinetic energy spectra (Figure 1(b)) are shown to be in close agreement with DNS apart for some slight underestimation of the closure transient field at the smallest scales. In Figure 1(c) the closure is shown to give a good estimate of the evolved Reynolds number throughout the evolution with $R_{L}^{\text {DNS }}(0.3)=164$ and $R_{L}^{\text {QDIA }}(0.3)=159$. In the second study at C48 resolution (Case II) the same initial conditions and parameters (but with $\triangle t=0.004$ ) are used; however, the topography now satisfies $\left|h_{\mathbf{k}}\right|^{2}=16 k^{2} /\left(1+k^{3}\right)^{2}$. This choice of topography falls away much more rapidly at the small scales than that used in the previous experiment thus resulting in a significantly reduced initial mean field kinetic energy at the small scales, as seen in Figure 1(d). An 
Case I
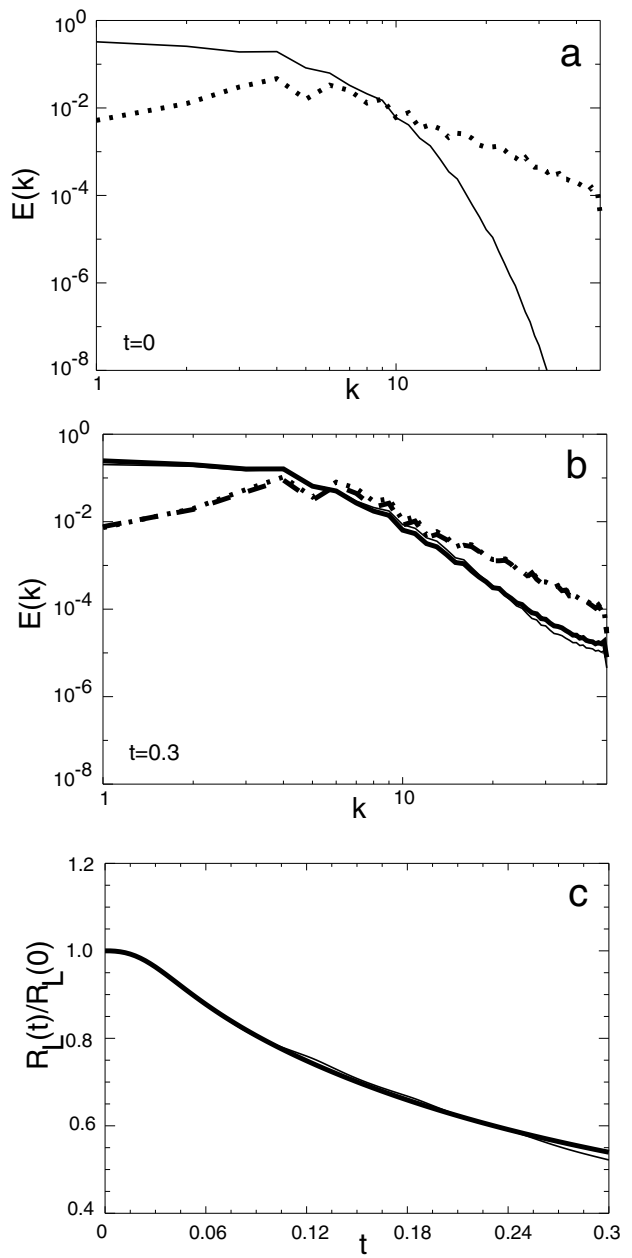

Case II
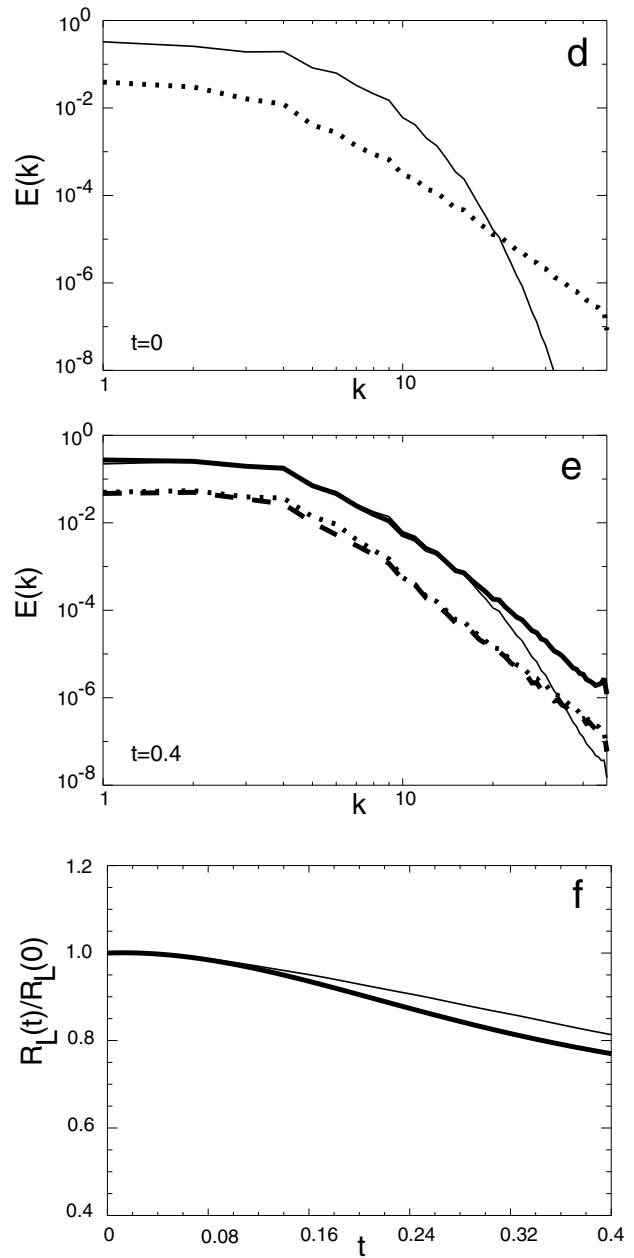

Figure 1: C48 resolution spectra. The initial mean and transient energy spectra are depicted in (a) and (d) with the final evolved energy shown in (b) and (e) respectively. The component field diagrams are as follows: DNS mean-field (dashed), QDIA mean-field (dotted), DNs transient-field (thick solid), QDIA transient-field (thin solid). The large-scale Reynolds number ((c) and (f)) are DNS (thick solid) and QDIA (thin solid). 
TABLE 1: Parameters

\begin{tabular}{llllll}
\hline Case & $\triangle \mathrm{t}$ & $\hat{\nu}$ & $a$ & $b$ & $\left|h_{\mathbf{k}}\right|^{2}$ \\
I & 0.003 & 0.0025 & $4.824 \times 10^{4}$ & $2.511 \times 10^{3}$ & $4 k /\left(1+k^{3}\right)$ \\
II & 0.004 & 0.0025 & $4.824 \times 10^{4}$ & $2.511 \times 10^{3}$ & $16 k^{2} /\left(1+k^{3}\right)^{2}$ \\
\hline
\end{tabular}

additional feature of a more rapidly decreasing topographic amplitude is a reduction in the strength of the eddy-topography interaction at the small scales. For $\left|h_{\mathbf{k}}\right|^{2}=16 k^{2} /\left(1+k^{3}\right)^{2}$ the evolved $\left(t_{f}=0.4\right)$ closure mean kinetic energy (Figure 1(e)) spectra show close agreement with DNS, as was the case when the topography falls away as $1 / k$. However, the transient energy spectra have undergone significant increases for $20<k<48$ after a period of evolution to $t_{f}=0.4$ as compared to the initial spectra; it is also evident that the QDIA gives good results in the energy containing range of large scales but underestimates the evolved small-scale transient energy. The failure of the closure to accurately predict the small-scale transient kinetic energy is not surprising given that we know spurious convection effects are inherent in the direct interaction approximation, due to the inaccurate treatment of the three-point cumulant term. These effects were found to be pronounced for this initial transient spectrum in the previous studies of Herring et al. [4] and Frederiksen \& Davies [7].

Figure 1(f) shows the evolution of $R_{L}(t) / R_{L}(0)$ for Case II. The DNS and QDIA closure large-scale Reynolds number are displayed and we note some increased discrepancy between the DNS and QDIA evolved Reynolds numbers for the Case II results as compared to Case I where we have a strong small scale topographic amplitude. The over-representation of the Reynolds number for Case II (Fig. 1 (f)) is a direct result of the closure under-representing the transients in the small scales. The initial Reynolds number for the Case II calculation is $R_{L}(0)=305$ with the evolved $R_{L}(0.4) / R_{L}(0)=0.77$ for the DNS calculation and $R_{L}(0.4) / R_{L}(0)=0.81$ for the QDIA. However, when the topographic strength at the small scales is increased the QDIA closure provides a better estimate of the large-scale Reynolds number (Figure 1(c)). 


\section{Discussion and Conclusions}

The QDIA has been demonstrated to very accurately capture the evolved kinetic energy in the large scales in both cases considered. In addition the QDIA compares well at all scales to DNS at moderate Reynolds number when the topographic and mean-field contributions are significant at small scales. For the case where the topographic amplitude falls away as $1 / k^{2}$ and the evolved small-scale transient field dominates, the closure is found to significantly under-represent the small-scale transients (see Figures 1(d) and (e)). This underestimation arises due to spurious convection effects inherent in the DIA treatment of the three-point terms. However, despite this, the closure meanfield compares very closely to DNS. Interestingly, the QDIA closure performs best when topographic amplitudes and mean fields are stronger, as evident in Case I (Figures 1(b) and (c)), while in perturbation theory they are assumed to be small prior to renormalization. Thus it appears that an increased topographic amplitude, combined with an increased initial mean field, in the small scales mitigates the tendency of the QDIA to generate spurious convection effects resulting from the lack of vertex renormalization. The energy containing large scales demonstrate very close agreement between DNS and closure in both cases in this study. The overall close comparison with DNS underscores the validity of the quasi-diagonal approximation. Most recently Frederiksen \& Davies [14] have tackled the spurious convection problem of the DIA using a heuristic vertex renormalization wherein the eddy interactions are localized using a cutoff parameter for the interaction coefficients (as suggested in [13]). In the future we plan to generalize the heuristic vertex renormalization or regularization method of Frederiksen \& Davies [14] to inhomogeneous flows in an effort to mitigate the spurious nonlocal eddyeddy interactions described in this present paper as well as possible nonlocal eddy-mean field and eddy-topography interactions.

Acknowledgments: TJO gratefully acknowledges the support of CSIRO Atmospheric Research and the School of Mathematics, Monash University. 
TJO also appreciates useful discussions with David J. Karoly.

\section{References}

[1] Kraichnan, R. H. 1959 The structure of isotropic turbulence at very high Reynolds numbers. J. Fluid Mech. 5, 497-543. C136, C141

[2] Martin, P. C., Siggia, E. D. \& Rose, H. A. 1973 Statistical dynamics of classical systems. Phys. Rev. A 8, 423-437. C136

[3] Kraichnan, R. H. 1964a Decay of isotropic turbulence in the direct interaction approximation. Phys. Fluids 7, 1030-1048. C136

[4] Herring, J. R., Orszag, S. A., Kraichnan, R. H. \& Fox, D. G. 1974 Decay of two-dimensional homogeneous turbulence. J. Fluid Mech. 66, 417-444. C136, C137, C142, C145

[5] Kraichnan, R. H. 1971 An almost-Markovian Galilean-invariant turbulence model. J. Fluid Mech. 47, 513-524. C137

[6] Frederiksen, J. S., Davies, A. G. \& Bell, R. C. 1994 Closure equations with non-Gaussian restarts for truncated two-dimensional turbulence. Phys. Fluids 6, 3153-3163. C138

[7] Frederiksen, J. S. \& Davies, A. G. 2000 Dynamics and spectra of cumulant update closures for two-dimensional turbulence.

Geophys. Astrophys. Fluid Dyn. 92, 197-231. C138, C141, C142, C143, C145

[8] Herring, J. R. 1977 On the statistical theory of two-dimensional topographic turbulence. J. Atmos. Sci. 34, 1731-1750. C137

[9] Holloway, G. 1978 A spectral theory of nonlinear barotropic motion above irregular topography. J. Phys. Oceanogr. 8, 414-427. C137 
[10] Kraichnan, R. H. 1972 Test-field model for inhomogeneous turbulence. J. Fluid Mech. 56, 287-304. C137

[11] Kraichnan, R. H. 1964b Diagonalizing approximation for inhomogeneous turbulence. Phys. Fluids 7, 1169-1177. C137

[12] Frederiksen, J. S. 1999 Subgrid-scale parameterizations of eddy-topographic force, eddy viscosity, and stochastic backscatter for flow over topography. J. Atmos. Sci. 56, 1481-1494. C137, C140

[13] Kraichnan, R. H. 1964c Kolmogorov's hypothesis and Eulerian turbulence theory. Phys. Fluids 7, 1723-1734. C138, C146

[14] Frederiksen, J. S. \& Davies, A. G. 2003 The regularized DIA closure for two-dimensional turbulence. Geophys. Astrophys. Fluid Dyn. (submitted). C138, C146

[15] Leith, C. E. 1971 Atmospheric predictability and two-dimensional turbulence. J. Atmos. Sci. 28, 145-161. C137, C138

[16] O'Kane, T. J. \& Frederiksen, J. S. 2003 Integro-differential closure equations for inhomogeneous turbulence. ANZIAM J. 44 (E), C569-C589. C137, C141

[17] Frederiksen, J. S. \& Sawford, B. L. 1980 Statistical dynamics of two-dimensional inviscid flow on a sphere. J. Atmos. Sci. 37, 717-732. C142 